\title{
Some New Properties of the Dilute Polymer Solutions II. Study of Thermodynamic Properties of Dilute Polymer Solutions by the Monte Carlo Method
}

\author{
A. S. Pavlov, P. G. Khalatur, and V. E. Eskin \\ Institute of Macromolecular Compounds of the Academy \\ of Sciences of the U.S.S.R., Leningrad, U.S.S.R.
}

(Received January 29, 1986)

\begin{abstract}
Computer simulation of dilute solutions of polymer chains has been carried out in the concentration range lower than the critical concentration of coil overlapping. For solvents of various thermodynamic strengths the intermolecular interaction of polymer coils was investigated. The concentration dependence of osmotic pressure $\pi=\pi(c)$ was calculated. It was shown that near the $\theta$-point the $\pi(c)$ curves can be non-monotonic even at very low concentrations as a result of multiparticle interactions. The results obtained make it possible to interpret some anomalies observed in the experimental study of light scattering and osmotic pressure of extremely dilute polymer solutions.
\end{abstract}

KEY WORDS Computer Simulation of Polymer Solutions / Intermolecular Interaction / Concentration Dependence / Some Anomalies of the $\pi(c)$ Curves /

At present, considerable progress in the understanding of equilibrium and dynamic properties of polymer solutions is being made. This is due to both the development of experimental methods of investigation and new theoretical approaches. ${ }^{1}$ It should be noted that the main effort is directed to the study of concentrated and "semi-dilute"solutions in which the concentration of the polymer, $c$, greatly exceeds the critical concentration of coil overlapping $c^{*} \simeq N / \bar{R}^{3}$ ( $N$ is the number of units in the chain and $\bar{R}$ is the average chain size). As regards dilute polymer solutions $\left(c \ll c^{*}\right)$, it is usually considered that their thermodynamic properties are adequately described by the classical theories based on the works of Zimm, Flory, Krigbaum etc. ${ }^{2-4}$ However, some data reported in the literature cannot be explained in the frame-work of classical concepts $^{5,6}$ in which a polymer coil is considered to be a cloud of independently distributed units. Thus, it has been found ${ }^{7,8}$ that at infinite dilution and in a thermodynamically good solvent, the probability of overlapping of approaching polymer coils is greatly different from zero, which contradicts the conclusions of the Flory-Krigbaum theory (FK). ${ }^{3,4}$ Moreover, it has been shown ${ }^{9}$ that the potential of the mean force of coil interaction obtaind in ref 4 (FK-potential) does not take into account quite correctly the dependence on the inter-coil distance and thermodynamic strength of the solvent. Interesting effects have also been found in the experimental study of very dilute polymer solutions by the light scattering method. ${ }^{10,11}$ The results obtained in ref 10 and 11 indicate that a "threshold" concentration limit $c_{0}\left(c_{0} \ll c^{*}\right)$ exists and below which, the experimental light scattering plot $c K / R_{0}=f_{1}(c)$ (where $R_{0}$ is the excess scattering intensity by $\theta$ angle and $K$ is the optical constant of the solution) and the related plot of osmotic pressure $\pi / c=f_{2}(c)$ have the quasihorizontal direction and at $c=c_{0}$ exhibits a 
kind of inflection. Thermodynamic considerations show, however, that the concentration dependences $c K / R_{0}$ and $\pi / c$ should be smooth functions without any inflections. This conclusion follows quite unequivocally from the analysis of virial expansion. Hence, the "disappearance" of concentration dependences $c K / R_{0}$ and $\pi / c$ at $c<c_{0}$ found in ref 10 and 11 seems actually an apparent effect due to the fact that the true shape of the curves cannot be reliably determined in the range of limiting low concentrations.

The main aim of this work is to calculate the concentration dependence of osmotic pressure at low $c$ with the aid of a computer according to the Monte Carlo method (MC). Let us have a system which consists of $n$ chains of $N$ unit in the volume $V$. If we mean by $c$ the volume fraction of units $\left(c=v_{0} n N / V, v_{0}\right.$-volume occupied by one unit), then the osmotic pressure $\pi$ is expressed ${ }^{12}$ by

$$
\pi / K T c_{\mathrm{p}}=1-\ln G(c)+\frac{1}{c} \int_{0}^{c} \ln G(c) \mathrm{d} c
$$

where $c_{\mathrm{p}}=n / V$ is the chain concentration, $K$ is Boltzmann's constant, $T$ is the absolute temperature, $G(c)=Z_{\mathrm{N}}^{(n)} / Z_{\mathrm{N}}^{(n-1)}, Z_{\mathrm{N}}^{(n)}$, and $Z_{\mathrm{N}}^{(n-1)}$ are the partition functions for the systems consisting of $n$ and $n-1$ interacting chains. The correlation function $G(c)$ was determined by the $\mathrm{MC}$ method from the relation

$$
G(c)=\frac{\sum_{i=1}^{\Omega} \exp \left\{-\beta\left[E_{i}^{(n-1)}+E_{i}^{(1)}+E_{i}^{(n-1,1)}\right]\right\}}{\sum_{i=1}^{\Omega} \exp \left\{-\beta\left[E_{i}^{(n-1)}+E_{i}^{(1)}\right]\right\}}
$$

where $\beta=1 / k T, E_{i}^{(1)}$ is the potential energy of the intrachains interactions in the $i$-th configuration, $E_{i}^{(n-1)}$ is the potential energy of the intra- and interchain interactions in the system of $n-1$ chains, $E_{i}^{(n-1,1)}$ is the energy that appears when one chain is added to the system including $(n-1)$ chains. All calculations were based on the model for a poly- ethylene chain described in ref 13 and refer to $T=300 \mathrm{~K}$.

The values of $E$ in eq 2 were calculated according to the potential of the " $6-12$ " type with the parameters $\varepsilon$ (depth of the hole in the $k T$-scale) and $\sigma=0.4 \mathrm{~nm}$ (equilibrium distance). When $r<d(d=0.3 \mathrm{~nm}$ is the hard diameter of the unit) it was supposed that $f(r)=\infty$ and this configuration was rejected. When $\varepsilon=0$, the properties of the good (athermal) solvent were imitated. The increase in $\varepsilon$ intensified the attraction of the units and corresponded to the deterioration of the solvent quality. The chains were generated by the MC method ${ }^{9,13}$ in the cubic cell with the periodic conditions and the length of the edge varying from 12 to $3333 d$.

The number $\Omega$ of independent configurations of the system consisting of one isolated chain and $(n-1)$ interacting chains determined and placed in the cell (volume $V$ ). Hence we have $\Omega$ terms in the sum of the denominator (eq 2). Subsequently for each $\Omega$ configurations an attempt was made to place randomly a separate chain into the cell already containing $(n-1)$ chains. If, after this procedure, there was no overlapping of units, the next term was added to the numerator of (eq 2). As a result, we obtained $\Omega^{*}\left(\Omega^{*} \leqslant \Omega\right)$ configurations for the system consisting of $n$ chains with a fixed concentration. In all calculations, it was assumed that $\Omega>2000$, and this ensured the accuracy for $G(c)$ about $8 \%$.

The number of the chains in the cell was varied from 2 to 6 . The number $N$ of units in the chain was usually 100 . All calculations were carried out on a BESM-6/7 computer.

It is clear from the results of calculation of the function $G(c)$ that at $c \rightarrow 0$ the function $G(c)$ tends to unity, i.e., at infinite dilution the coils do not affect one another, as was to be expected. At $c>0$ in a good solvent (at low $\varepsilon$ values) $G(c) \leqslant 1$. If the attraction of units is sufficiently strong $(\varepsilon \gtrsim 0.06)$, then some values of $G(c) \geqslant 1$ appear. It should be noted that the inequality $G(c)<1$ means that the addition of 
another chain into the system (i.e., an increase in its concentration) is thermodynamically unfavorable. In contrast, in the case $G(c)>1$, this addition is preferable because the free energy of the system decreases. However, at the investigated values of the parameter $\varepsilon$ the free energy gain is observed only in the range of highly dilute solutions at $c \leqslant 0.03$ which is lower than $c^{*}$ (for the 100-unit chain model considered here $\left.c^{*} \approx 0.037^{9}\right)$. With increasing $c$ at $c>c^{*}$, the function $G(c)$ acquires much lower values than unity.

In the determination of $\pi$ according to eq 1 it was found necessary to carry out a preliminary approximation of the calculated values of $G(c)$ by a smooth function. For this purpose, on the basis of theoretical considerations, ${ }^{14} G(c)$ was represented by $G(c)=a_{1}$ $\exp \left(a_{2} c\right)+a_{3} \exp \left(a_{4} c^{2}\right)$ where the parameters $a_{1}-a_{4}$ were determined by the method of multiparametric optimization. ${ }^{15}$ After this integration necessary according to (1) was carried out, the results were approximated by means of the polynomials of the second degree:

$$
\begin{aligned}
& \left(\pi / c_{\mathrm{p}}\right) k T=1+A_{2}^{*} c+A_{3}^{*} c^{2} \\
& \lim _{\theta \rightarrow 0}\left(c_{\mathrm{p}} M^{2} K / R_{\theta} v_{0}\right)=1+2 A_{2}^{*} c+3 A_{3}^{*} c_{2}
\end{aligned}
$$

These relations following from the virial expansion -(here the values of $A_{k}^{*}=A_{k} M^{k}$ $\left(c_{\mathrm{p}} / v_{\mathrm{p}}\right)^{k-1}$ and $A_{k}$ are virial coefficients and $M$ is the molecular weight of the chain). Figure 1 shows the value of $\pi^{*}(c)=\left(\pi / c_{\mathrm{p}}\right) k T-1$ $v s$. $v c$ obtained for various values of $c$ and $\varepsilon$. The shape of the curve $R^{*}(c)=\left(c_{\mathrm{p}} M^{2} K /\right.$ $\left.R_{0} v_{0}-1\right)_{\theta=0}$ is quite similar to this dependence.

The two-parameter eq 3 and 4 adequately approximate the results of the calculation at $c<c^{*}$. This fact confirms the possibility of using the van der Waals equation, which is also two-parametric. It should be noted that the model for a dilute polymer solution based on the van der Waals state equation has been proposed in ref 11 and 16.
Before considering the functions $\pi^{*}(c)$ and $R^{*}(c)$, a value more convenient than the parameter $\varepsilon$ will be introduced for the characterization of the strength of the solvent. It is natural to express this property in the terms of the swelling coefficient $\alpha=\left(\left\langle R^{2}\right\rangle /\right.$ $\left.\left\langle R_{\theta}^{2}\right\rangle\right)^{1 / 2}$ calculated for an isolated coil $(c \rightarrow 0)$. In this case, the average dimensions of a chain with volume interactions are obtained by the Monte Carlo method for the predetermined $\varepsilon$, whereas the values of $\left\langle R_{\theta}^{2}\right\rangle^{1 / 2}$ representing the mean-square dimensions of an ideal (unperturbed) chain can be obtained by a precise analytical method. ${ }^{17}$ Figure 2 shows the dependence of $\left(\alpha^{2}-1\right)$ on $\varepsilon$ for chains of various lengths. The condition $\alpha^{2}=1$ determines the values of $\varepsilon_{\theta}$ when coil swelling disappears and the conditions of the $\theta$-solvent are simulated. It was found that for the $N$ values considered an explicit dependence of $\varepsilon_{\theta}$ on $N$ exists. The values of $\varepsilon_{\theta}(N)$ are represented as a function of the argument $N^{-1}$ in Figure 3. Treatment of results by the least-squares method in the range of $N=40-400$ gave $\varepsilon_{\theta}(N)=0.114-2.82 / N$. Hence, at $N \rightarrow \infty$ for the model investigated, $\varepsilon_{\theta}=0.114$. Thus, at $\varepsilon=\varepsilon_{\theta}$, finite chains are compressed with respect to their $\theta$-dimensions. On the other hand, it is possible to say that at $\varepsilon \leqslant 0.1$ the conditions of a good solvent are essentially simulated for the isolated coils considered here if the solvent strength is evaluated from the position of the "true" $\theta$-point corresponding to $\varepsilon_{\theta}=0.114$.

Now we will consider intermolecular effects. Figure 1 shows that if repulsion forces between units predominate $(\varepsilon \leqslant 0.06)$, the value of $\pi^{*}(c)$ increases monotonically with increasing $c$. For the range of $c \lesssim 1.5 \times 10^{-2}$, the function $\pi(c)$ is almost linear, which indicates the predominant part played by binary interactions of polymer coils in a dilute solution. At high $c$, the multiparticle (ternary, etc.) interactions of polymer coils begin to be observed. This leads to the deviation of the dependence $\pi^{*}(c)$ from a linear dependence. 


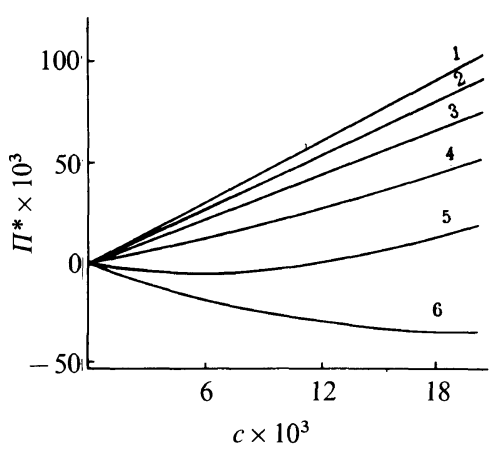

Figure 1. Dependence of $\Pi^{*}$ on $c$ at $\left.\varepsilon=1\right) 0$;2) 0.02 ; 3) 0.04 ; 4) 0.06 ; 5) 0.08 ; 6) 0.10 .

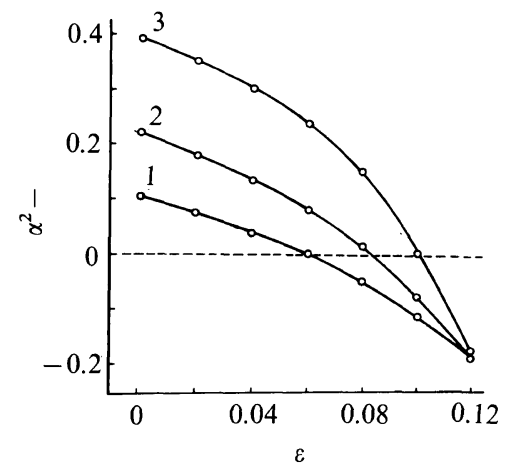

Figure 2. Dependence of $\alpha^{2}-1$ on $\varepsilon$ for $N=1$ ) 50,2 ) 100 and 3) 200.

It is characteristic that although the coils are compressed, the contribution of multiparticle interactions increases with decreasing solvent strength. Thus, at $c=1.5 \times 10^{-2}$ and $\varepsilon=0$ the contribution of the term $\left(A_{3}^{*} c^{2}\right)$ to the overall value of $\pi^{*}(c)$ is $\approx 1 \%$, whereas at $\varepsilon=0.06$ and at the same concentration, this contribution increases to $20 \%$. This fact can be explained as follows: the increase in $\varepsilon$ leads to coil compression (Figure 2) and an increase in the distance at which their interaction begins to appear.

Moreover, since the repulsive part of the potential of the mean force of coil interaction is flat, ${ }^{7-9}$ if a relatively strong attraction exists, the coils should exhibit a much more pronounced trend towards the formation of clusters (intermolecular aggregates) than

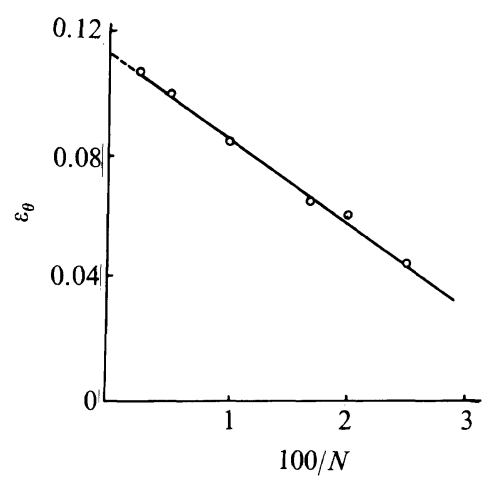

Figure 3. Dependence of $\varepsilon_{\theta}(N)$ on the inverse value of the number of units in a polymer chain.

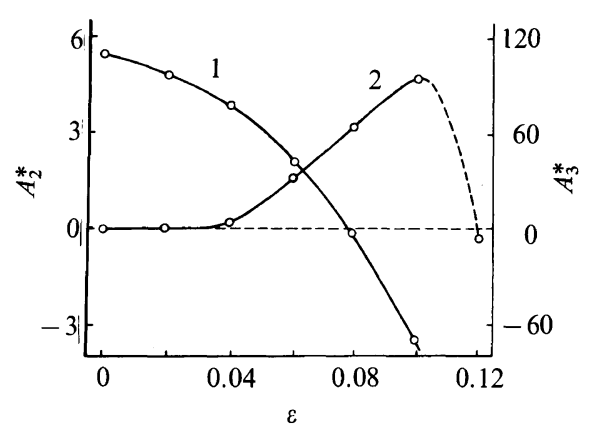

Figure 4. Dependence of 1) $A_{2}^{*}$ and 2) $A_{3}^{*}$ on parameter

small molecules. The authors will consider this problem again in a separate publication.

Figure 4 shows the dependence of $A_{2}^{*}$ and $A_{3}^{*}$ on $\varepsilon$. Taking into consideration the fact that the errors in calculation increase greatly at $\varepsilon \gtrsim 0.12$, the corresponding results in the range of $\varepsilon>0.1$ should be considered only as tentative. It is noteworthy that the general shape of the curves $A_{2}^{*}(\varepsilon)$ and $A_{3}^{*}(\varepsilon)$ obtained for polymer coils coincides with that characteristic of a gas consisting of the Lennard-Jones particles. Figure 4 also shows that the inversion of the sign of $A_{2}^{*}$ occurs at a slightly lower value of $\varepsilon$ than $\varepsilon_{\theta}$. Alternatively, at $A_{2}^{*} \lesssim 0$ the coils remain slightly swollen. Hence, by using for finite $N$ various methods of the determination of the $\theta$-point $(\alpha=1$, $A_{2}=0$, etc.) we obtain various values of the $\theta$ temperature (or the parameter $\varepsilon$ ). Hence, at $N^{-1}>0$ there is no single $\theta$-point at which all 
the properties of a real system coincide with those of an ideal system but a certain finite $\theta$ range exists. It should be noted that the concept of the $\theta$-range has been first introduced in ref 18 and 19. At $N \rightarrow \infty$ the $\theta$-range contacts the $\theta$-point. ${ }^{19}$

The behavior of curves 5 and 6 (at $\varepsilon \geqslant 0.08$ ) in Figure 1 is similar to that of experimental dependences obtained for benzene solutions of polystyrene samples with the number of repeat units $N \sim 100 .^{10,11}$ At $c<c_{0}$ where $c_{0}=-A_{2}^{*} / A_{3}^{*}$ the value of $\pi^{*}(c)$ is negative (Figure 1). The maximum inflection of the curve equal to $\pi_{\min }^{*}=-\left(A_{2}^{*}\right)^{2} / 4 A_{3}^{*}$ at $c=c_{0} / 2$ if very small and hence is very indistinguishable. This is probably the reason for the apparent "disappearance" of the dependence of $\pi^{*}$ and $R_{\theta}^{*}$ on $c$ at $c<c_{0}{ }^{10,11}$

Unfortunately, since computer simulation is a very laborious procedure, it was impossible to study the molecular-weight dependences (we intend to do so in another paper). However, some rough evaluations can be made by using the concepts of the scaling theory. According to ref 1 , the dependence of the $k$-th virial coefficient on $N$ is expressed as $A_{k} \sim N^{k(1-\eta)-2+\eta}$, where $\eta$ is the critical index close to $1 / 5$. Hence, at $N \gg 1$ the third virial approximation gives $c_{0} \sim N^{\eta-1}$ and $\pi_{\mathrm{min}}^{*} \sim$ const, i.e. the maximum inflection on the curve of osmotic pressure should be independent of $N$ (or $M$ ). It should be emphasized that in these evaluations, the identical relationship is assumed between $A_{k}$ and $N$ both at $T>\theta$ and near the $\theta$-point at the inversion of the sign of $A_{2}$. The dependence of $c_{0} \sim N^{\eta-1} \sim N^{-4 / 5}$ coincides with that of $c^{*}$ on $N$. However, computer simulation shows that $c_{0} \ll c^{*}$. This is in agreement with experimental data. ${ }^{10,11}$

Now some conclusions may be formulated. The behavior of osmotic pressure (and hence of the function of light scattering $R^{*}(c)$ ) in the range of limiting low concentrations at $c \rightarrow 0$ is, naturally, determined only by the value and sign of the second virial coefficient. A complicating feature for solutions of chains of finite length is the existence of a very wide $\theta$-range in which various characteristics $\left(\alpha, A_{2}, A_{3}, \ldots\right)$ acquire "ideal" values at different $T$ (or $\varepsilon$ ). For short chains the $\theta$ range may also cover the conditions under which long chains exhibit considerable swelling and which from this standpoint may be regarded as conditions corresponding to a good solvent. Moreover, as shown above, near the $\theta$-point, even at very low concentrations, the multiparticle interactions of coils begin to be appreciable. It is the "addition" of these coils that leads to the non-monotonic dependences of $\pi^{*}(c)$ and $R^{*}(c)$ (see Figure 1). However, this effect may be very weak and, as already indicated, its experimental detection is difficult. This may cause many artifacts. Thus, if the experimental investigations of short-chain solutions $(N \sim 100)$ are limited to the observations in the range $c \gtrsim c_{0}$, then extrapolation of the curves $\pi(c)$ or $c K / R_{\theta}$ to $c=0$ can give both excessively high values of molecular weight of the chain and excessively high values (and even the wrong sign) of the second virial coefficient. The latter fact will evidently lead to erroneous evaluation of the thermodynamic strength of the solvent. On the other hand, investigations in the range of $c \lesssim c_{0}$ for solutions of chains of low molecular weight involve considerable technical difficulties, and at $c<c_{0}$ it is impossible to determine the true shape of the curves (similar to curve 5 in Figure 1). In this case, the scattering of experimental points leads to the above mentioned "disappearance" of concentration dependence of the $c K / R_{\theta}$ value. ${ }^{10,11,16}$

Acknowledgment. The authors wish to express their gratitude to A. M. Eljashevitch and to A. R. Khokhlov for their helpful comments.

\section{REFERENCES}

1. P.-G. de Gennes, "Scaling Concepts in Polymer 
Physics," Cornell Univ. Press, Ithaca London, 1979.

2. B. H. J. Zimm, Chem. Phys., 14, 146 (1946).

3. P. J. Flory, J. Chem. Phys., 17, 1347 (1949).

4. P. J. Flory, W. R. Krigbaum, J. Chem. Phys., 18, 1086 (1950).

5. P. J. Flory, "Principles of Polymer Chemistry," Cornell University Press, Ithaca, New York, 1953.

6. H. Yamakawa, "Modern Theory of Polymer Solutions," Harper \& Row, New York, N. Y., 1971.

7. P. G. Khalatur and A. R. Khokhlov Dokl. Akad. Nauk SSSR, 259, 1357 (1981).

8. A. Yu. Grosberg, P. G. Khalatur, and A. R. Khokhlov, Makromol. Chem. Rapid Commun., 3, 709 (1982).

9. P. G. Khalatur and Yu. G. Papulov, "Mashinny eksperiment," V. Konformatsionnomanalizepolimerov, Kalinin, Kalinin University, 1982.

10. V. E. Eskin and Y. A. Baranovskaya, Vysokomol.
Soedin. Ser. A, 19, 5337 (1977).

11. V. E. Eskin and Y. A. Baranovskaya, Vysokomol. Soedin. Ser. A, 24, 2549 (1982).

12. H. Okamoto, A. Bellemans, J. Phys. Soc. Jpn., 47, 955 (1979).

13. P. G. Khalatur, Vysokomol. Soedin. Ser. A, 21, 2687 (1979).

14. R. J. Koyama, Chem. Phys., 72, 247 (1980).

15. D. M. Himmelblau, "Applied Nonlinear Programming," McGraw-Hill, New York, N. Y., 1972.

16. V. E. Eskin, Vysokomol. Soedin. Ser. B, 23, 725 (1981).

17. P. J. Flory, "Statistical Mechanics of Chain Molecules," Interscience, New York, N. Y., 1969.

18. H. A. C. Chermin and J. W. Kennedy, Macromolecules, 5, 655 (1972).

19. A. R. Khokhlov, J. Phys., 38, 845 (1977). 